\title{
Evaluación de las Tasas de Supervivencia Clínica de los Implantes Cortos. Revisión de la Literatura
}

\author{
Evaluation of Clinical Survival Success of Short Implants. Review of the Literature
}

\author{
Adrián Curto Aguilera
}

\begin{abstract}
CURTO, A. A. Evaluación de las tasas de supervivencia clínica de los implantes cortos. Revisión de la literatura. Int. J. Odontostomat., 6(2):201-203, 2012.

RESUMEN: El objetivo de esta investigación fue evaluar las diferentes publicaciones de estudios clínicos sobre el empleo de implantes cortos y su éxito clínico. Se realizó una revisión sistemática en Enero de 2012 en la base de datos PubMed y SciELO con las palabras clave: dental implants, short dental implants y success survival. Se evaluaron los artículos publicados en los últimos cinco años. Los resultados se limitaron a los ensayos clínicos. Los artículos relevantes fueron analizados. Cuatro artículos participaron en la revisión final. Se colocaron un total de 4300 implantes de los que fracasaron 41 (0,95\%). Se encontraron tasas de supervivencia del 96,6\% hasta el 99,3\%. Los estudios recientes muestran que los implantes cortos pueden ser predecibles y que tienen tasas de éxito similares a las de implantes convencionales siempre que sean utilizados bajo una cuidadosa planificación de tratamiento y protocolos estrictos.
\end{abstract}

PALABRAS CLAVE: implantes dentales, implantes cortos, tasas de supervivencia.

\section{INTRODUCCIÓN}

Los protocolos rehabilitadores prostodónticos han ido evolucionando en los últimos años gracias a los avances en los procedimientos de cirugía implantológica, al igual el desarrollo de los materiales que conforman los implantes.

En las últimas décadas el éxito y la predictibilidad a largo plazo de la osteointegración en relación a la colocación de implantes dentales ha generalizado las rehabilitaciones prostodónticas implantosoportadas, tanto en los casos de edentulismo parcial como total, siendo, en la gran mayoría de casos el tratamiento de primera elección.

Debido al gran auge de la implantología, es cada vez más frecuente encontrarse casos complejos donde debido a las condiciones de los pacientes, presentan un grado severo de reabsorción alveolar donde la colocación de implantes es a menudo problemática y suele requerir cirugías adicionales para aumentar el nivel óseo. Esto ocurre generalmente en los sectores posteriores maxilares y mandibulares donde existe el riesgo de afectar al nervio alveolar inferior o perforar el seno maxilar durante la colocación de los implan- tes, aumentando la morbilidad quirúrgica en estos procedimientos junto con las complicaciones postoperatorias. Además las zonas posteriores presentan mayor dificultad para la colocación de implantes (acceso difícil, visibilidad limitada, espacio reducido, pobre calidad ósea y mayores cargas oclusales).

Una alternativa predecible para compensar la escasez de altura ósea adecuada es el uso de diferentes técnicas quirúrgicas que faciliten el aumento óseo, como la elevación de seno maxilar, la regeneración ósea guiada, la distracción ósea, etc, que aumentan la morbilidad postquirúrgica y el costo total, así como la duración del tratamiento, lo que muchas veces se traduce en que el paciente rechace esta opción (Kotsovilis et al., 2009; Anitua et al., 2008; Morand \& Irinakis, 2007).

Otra opción es el uso de implantes cortos, sin embargo éstos han estado tradicionalmente relacionados con tasas de supervivencia más bajas en comparación con los implantes de longitud convencional. Se afirmaba que el uso de los implantes una mayor longitud proporcionaba mayores ventajas clínicas, debido a una 
mejor proporción corono-radicular y a una mayor superficie implantaría $y$, por tanto, mayor área de osteointegración.

Los estudios actuales indican que se puede alcanzar la misma tasa de éxito clínico con implantes cortos si se compara con los de mayor longitud, siendo su uso una alternativa terapéutica predecible a corto y medio plazo (Fugazzotto, 2008; Maló et al., 2007).

Es necesario definir el término "implante corto". Algunos autores los han definido como aquellos que no superan más de $7 \mathrm{~mm}$ de longitud. Se considera como implante corto a aquellos de 6 a $8 \mathrm{~mm}$ de longitud e implantes convencionales de 10 hasta $16 \mathrm{~mm}$ de longitud (Arlin, 2006).

Diversos ensayos clínicos demuestran que la predictibilidad de los implantes cortos es comparable a la de los implantes convencionales, es decir, de longitud normal. Afirman que el máximo estrés óseo es prácticamente independiente de la longitud del implante. Es más importante el diámetro del implante que la longitud del mismo. A nivel biomecánico reducir al máximo las fuerzas oclusales laterales en las prótesis implantosoportadas, así como distribuir uniformemente estas fuerzas mediante el uso de implantes ferulizados juega un papel significativo en la disminución del estrés sobre los implantes cortos (Anitua \& Orive, 2010).

El fracaso de un implante dental ha sido asociado a diversos factores como: pobre calidad ósea, longitud corta, diámetro estrecho, sexo, infecciones, área de implantación y hábitos parafuncionales. Algunos de estos factores pueden ser más importantes que otros, pero la mayor complicación reside en la asociación de dos o más factores (das Neves et al., 2006).

\section{MATERIAL Y MÉTODO}

Se realizó una revisión bibliográfica de los artículos indexados en las base de datos PubMed y SciELO sobre aquellos estudios publicados en los últimos cinco años, hasta Enero de 2012. Como criterio principal de inclusión se estableció que los estudios fueran ensayos clínicos excluyendo las revisiones sistemáticas de casos. Se emplearon las palabras clave: dental implants, short dental implants y success survival. Se encontraron cuatro artículos que fueran ensayos clínicos y que, por tanto, cumplían el criterio de inclusión.

\section{RESULTADOS}

Los artículos de ensayos clínicos analizados fueron un total de cuatro. Todos los artículos son estudios retrospectivos y comparan diferentes sistemas de implantes (BTI, Straumann y Branemark).

Los resultados han sido recogidos en la Tabla I de acuerdo al número de pacientes evaluados, edad de los mismos, seguimiento, tipo de implante colocado, número de implantes evaluados, longitud y diámetro de los mismos, localización, número de fracasos y tasa de supervivencia.

La suma total de implantes colocados fue de 4300. De ellos fracasaron $41(0,95 \%)$. La tasa de supervivencia media fue de $98,53 \%$, la mínima fue de $96,6 \%$ y la máxima de $99,3 \%$. La edad media de los pacientes evaluados fue de 56 años, siendo la edad mínima 20 años y la máxima 94. 62,375 meses fue el tiempo medio de seguimiento, abarcando un periodo mínimo de 1 hasta 108 meses. La longitud media de los implantes colocados fue de $7,7 \mathrm{~mm}$, abarcando implantes desde $6,5 \mathrm{~mm}$ de longitud hasta implantes de $8,9 \mathrm{~mm}$. El diámetro medio de los implantes fue de $4,39 \mathrm{~mm}$, siendo el diámetro mínimo $2,5 \mathrm{~mm}$ y el máximo $6,5 \mathrm{~mm}$. $1738(40,42 \%)$ implantes fueron colocados en el maxilar y $2562(59,58 \%)$ en la mandíbula. 10,25 implantes fue el número de fracasos medio.

\section{DISCUSIÓN}

En general la elección de la longitud de los implantes dentales está en relación a la cantidad y calidad del hueso basal disponible. Esto se basa en que los implantes más largos proporcionan una mejor estabilidad primaria en el lecho periimplantario y una distribución de las fuerzas oclusales más favorables y adecuadas como consecuencia de la mayor superficie del implante dental (Raviv et al., 2010).

Es necesario la realización de estudios clínicos con un seguimiento a largo plazo que permitan evaluar la evolución clínica en el uso de implantes cortos.

Los estudios recientes muestran que los implantes cortos pueden ser predecibles y que tienen tasas de éxito similares a las de los implantes convencionales, siempre que sean utilizados bajo una cuidadosa planificación de tratamiento. 


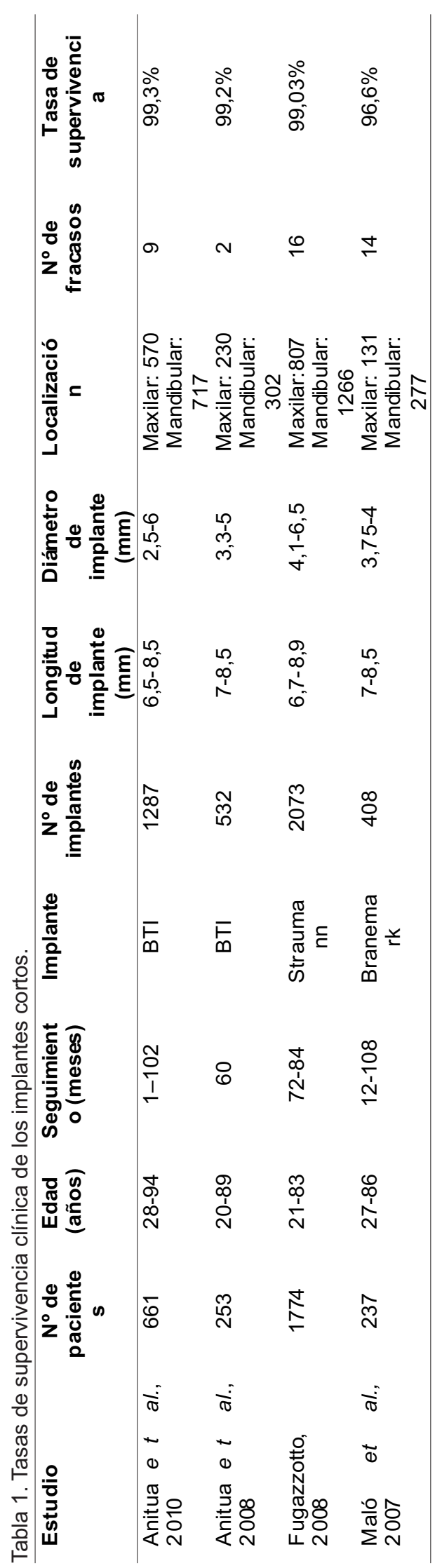

CURTO, A. A. Evaluation of clinical survival success of short implants. Review of the literature. Int. J. Odontostomat., 6(2):201-203, 2012.

ABSTRACT: The purpose of this study was to evaluate different published clinical studies of short dental implants and their clinical success rate. A systematic search in January 2012 in PubMed and SciELO database with key words: dental implants, short dental implants and success survival. We evaluated the articles published in the last five years. Results were limited to clinical trials. Relevant articles were then evaluated. Four articles entered the final review. A total of 4300 implants were placed of which $41(0.95 \%)$ failed. The survival rates ranged from $96.6 \%$ to $99.3 \%$. Recent studies demostrate that short dental implants can be predictable and have a success rate similar to that of conventional implants as long as they are placed after careful planning and following strict protocol.

KEYWORDS: dental implants, short implants, success survival.

\section{REFERENCIAS BIBLIOGRÁFICAS}

Anitua, E. \& Orive, G. Short implants in maxillae and mandibles: a retrospective study with 1 to 8 years of follow-up. J. Periodontol., 81(6):819-26, 2010.

Anitua, E.; Orive, G.; Aguirre, J. J. \& Andía, I. Five-year clinical evaluation of short dental implants placed in posterior areas: a retrospective study. $J$. Periodontol., 79(1):42-8, 2008.

Arlin, M. L. Short dental implants as a treatment option: results from an observational study in a single private practice. Int. J. Oral Maxillofac. Implants, 21(5):769-76, 2006.

das Neves, F. D.; Fones, D.; Bernardes, S. R.; do Prado, C. J. \& Neto, A. J. Short implants--an analysis of longitudinal studies. Int. J. Oral Maxillofac. Implants, 21(1):86-93, 2006.

Fugazzotto, P. A. Shorter implants in clinical practice: rationale and treatment results. Int. J. Oral Maxillofac. Implants, 23(3):487-96, 2008.

Kotsovilis, S.; Fourmousis, I.; Karoussis, I. K. \& Bamia, C. A systematic review and meta-analysis on the effect of implant length on the survival of roughsurface dental implants. J. Periodontol., 80(11):1700-18, 2009.

Maló, P.; de Araújo Nobre, M. \& Rangert, B. Short implants placed one-stage in maxillae and mandibles: a retrospective clinical study with 1 to 9 years of follow-up. Clin. Implant Dent. Relat. Res., 9(1):15-21, 2007.

Morand, M. \& Irinakis, T. The challenge of implant therapy in the posterior maxilla: providing a rationale for the use of short implants. J. Oral Implantol., 33(5):257-66, 2007.

Raviv, E.; Turcotte, A. \& Harel-Raviv, M. Short dental implants in reduced alveolar bone height. Quintessence Int., 41(7):575-9, 2010.

Dirección para correspondencia:

Adrián Curto Aguilera

C/Eras, 4.

37710. Candelario

Salamanca - ESPAÑA

Email: adriancurtoaguilera@gmail.com

Recibido : 12-02-2012 Aceptado: 12-07-2012 